1. Sedgwick, B. Nat. Rev. Mol. Cell Biol. 5, 148-157 (2004).

2. Hickman, M.J. \& Samson, L.D. Proc. Natl. Acad. Sci. USA 96, 10764-10769 (1999).

3. Hickman, M.J. \& Samson, L.D. Mol. Cell 14 105-116 (2004)
4. Rasimas, J.J., Dalessio, P.A., Ropson, I.J., Pegg, A.E. \& Fried, M.G. Protein Sci. 13, 301-305 (2004).

5. Teo, A.K., Oh, H.K., Ali, R.B. \& Li, B.F. Mol. Cell. Biol. 21, 7105-7114 (2001).

6. Ali, R.B. et al. Mol. Cell. Biol. 18, 1660-1669 (1998).
7. Daniels, D.S. et al. EMBO J. 19, 1719-1730
(2000).
8. Daniels, D.S. et al. Nat. Struct. Mol. Biol. 11,
$714-720$ (2004).
9. Rasimas, J.J., Pegg, A.E. \& Fried, M.G. J. Biol. Chem.
$278,7973-7980(2003)$.

\title{
Recognizing a phosphate group
}

The addition of a phosphate group (four atoms in total) to a protein can significantly alter its function by modulating its catalytic activity, inducing a conformational change or affecting its interactions with other proteins or nucleic acids. For example, in transcription, phosphorylation of the C-terminal domain (CTD) of RNA polymerase II, the enzyme that synthesizes messenger RNA (mRNA), modulates both transcription and mRNA processing. How the various transcription and processing factors recognize and distinguish between the phosphorylated and unphosphorylated polymerase tail is not well understood.

The Pol II CTD is located near the RNA exit channel, and consists of up to 52 repeats of the sequence Tyr1-Ser2-Pro3Thr4-Ser5-Pro6-Ser7. Phosphorylation at Ser2 and Ser5 regulates transcription and the binding of mRNA capping, splicing and polyadenylation factors. Specifically, during transcription initiation, the CTD becomes phosphorylated at Ser5. This modification initiates the binding of the $5^{\prime}$-capping enzymes for the first step of RNA processing. Phosphorylation at Ser2 accompanies transcription elongation and is required for processing of the $3^{\prime}$ end of the mRNA. Several RNA-processing factors recognize the CTD through a conserved CTD-interacting domain (CID). How the CID distinguishes between a phosphorylated and non-phosphorylated CTD is not clear. Meinhart and Cramer (Nature 428, 223-226; 2004) determined the structure of CID of yeast Pcf11, an essential protein for processing of the $3^{\prime}$ end of the mRNA and for transcription termination, bound to Ser2-phosphorylated CTD peptide. Surprisingly, the phosphate group does not make direct contacts with the protein, which suggests that the modification is recognized indirectly by the CID.

The CID of Pcf11 consists of $\alpha$-helices arranged in a righthanded superhelix resembling the folds in VHS domains and armadillo-repeat proteins. The CTD peptide (yellow sticks) binds in a groove lined with conserved residues (green surface). The central residues of the CTD peptide adopt a $\beta$-turn. Previous NMR studies of similar peptides that were unphosphorylated identified possible turn structures in the vicinity of Ser2 indicating that the peptide does not require the phosphorylation of Ser2 to form the $\beta$-turn. These data suggest that the structure observed in the CID-CTD peptide complex is not likely the result of induced fit.

The phosphate group (red sticks) of Ser2 is solvent exposed and points away from the CID surface. The lack of direct contacts between the phosphate group and the protein explains why Pcf11 can also bind unphosphorylated CTD. However, phosphorylation of Ser2 strongly enhances CTD binding, so the protein is able to indirectly distinguish between the two modification states. How might this occur? In the unphosphorylated state, the $\beta$-turn is stabilized by two hydrogen bonds, between Ser2 and Thr4 and Ser5. When Ser2 is modified, the phosphate group forms an additional hydrogen bond between this residue and the hydroxyl of Thr4. Meinhart and Cramer propose that the stabilization of the $\beta$-turn by this additional intramolecular hydrogen bond is important for the indirect recognition of the phosphorylated peptide. How the CID discerns the stability of the CTD peptide is not clear and will require additional studies.

Comparison of this CTD structure with that of Ser5-phosphorylated peptide bound to the Cgt1 subunit of the capping enzyme and to that of the Ser2/Ser5-phosphorylated peptide bound to the Pin 1 prolyl isomerase indicates that the CTD can adopt different conformations depending on its binding partner. Phosphorylation of either Ser 2 or Ser 5 could remodel any of the 52 repeats in the CTD and thereby modulates their interactions with a diverse group of proteins during transcription and mRNA processing.

Evelyn Jabri 